\title{
An Algorithm for Fast Corner Points Extraction with Reducing Mistaken Matching Based on Harris Operator
}

\author{
Xiaoyuan Chen ${ }^{1, a, *}$, Yong Zhu ${ }^{2, b}$, Bo Xiao ${ }^{2, c}$, Yuqiang Dai ${ }^{1, d}$, Jin Duan ${ }^{2, e}$ \\ ${ }^{1}$ School of Electronic and Information Engineering,Changchun University of Science and \\ Technology,Changchun,JiLin 130022,China \\ ${ }^{2}$ School of Computer Science and Technology,Changchun University of Science and \\ Technology,Changchun,JiLin 130022,China \\ a946201220@qq.com, bzhy991112@sina.com , c706992535@qq.com, d846250861@qq.com, \\ eduanjin@vip.sina.com \\ *corresponding author : zhy991112@sina.com
}

\begin{abstract}
The paper proposes a new methods which used in image matching Harris corner extraction and corner matching. On the basis of the original Harris algorithm, the efficiency of extracting corners is improved by reducing the number of computing the corner response value. Then the NCC algorithm is used to match the extracted corners, and the matching accuracy is improved by choosing the threshold (auto correlation coefficient). The experimental results show that the proposed algorithm reduces the time of corner extraction, and is superior to the traditional Harris corner extraction method. Moreover, when matching the two images with NCC matching algorithm, the mismatching is reduced. Finally, the Ransac algorithm is used to remove the false matches.
\end{abstract}

Keywords: Harris operator, corner detection, NCC algorithm, Ransac algorithm.

\section{Introduction}

The corners of the image contain a lot of information, and the number of corners is much smaller than the number of pixels, but the corner can be able to express the object shape, size and other important information [1]. It retains the image of the important characteristics of the object information at the same time effectively reduce the amount of information data . In the computer vision and image processing, there are different definitions of corner points ; Corner points can be defined as the point where the gray scale of the local area changes drastically, the curve or the intersection of the straight line, the curvature of the target area edge curve and so on ${ }^{[2]}$. At present, there are two kinds of corner detection methods ${ }^{[3]}$. First, based on the image contour edge of the method, this algorithm in the corner detection on the edge line dependence is relatively large, and sensitive to noise, if the edge line interrupt, Will have an impact on the results. Second, based on the image gray-scale method, mainly based on the field of gray-scale changes in the corner point to determine the location. The most famous is the Harris algorithm, because Harris algorithm corner detection has a better result, it is calculated by the location of the pixel gradient detection point ${ }^{[4]}$.

\section{Experimental flow chart}

According to the structure diagram, two images are selected as the original image and the contrast image. The traditional Harris algorithm and the improved algorithm are used to extract the corners of the two images, and then the NCC algorithm is used to match the corner points. Which is the use 
of the original NCC matching algorithm for the corner of the match, the solid line is improved by using the NCC matching algorithm for coarse angle matching, and finally use the Ransac algorithm for fine matching, The matching rate is calculated by these two methods, At last compare the two methods. The flow chart is as follows:

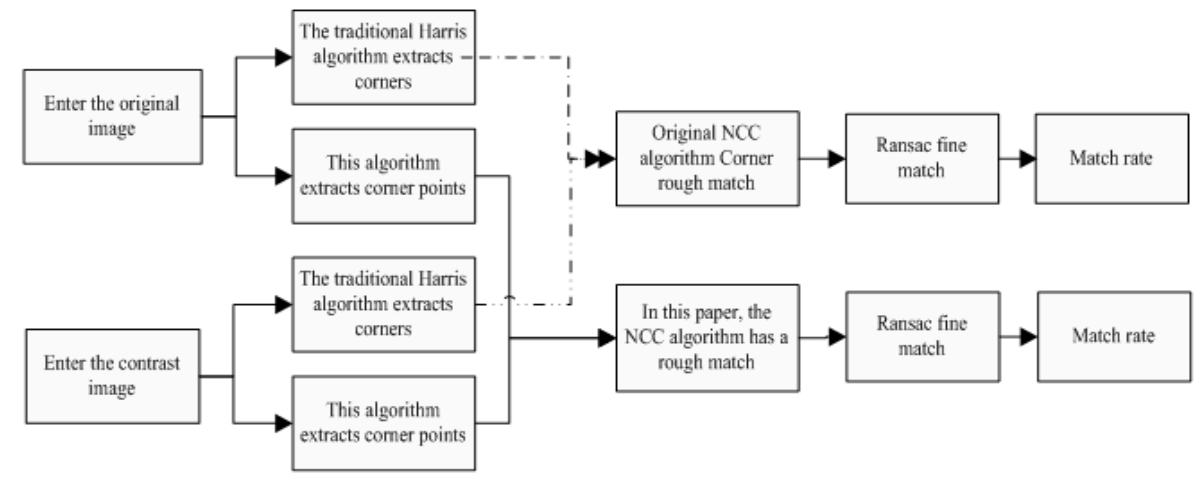

Fig.1 Experimental flow chart

\subsection{Harris algorithm principle}

Harris corner detection algorithm is a signal-based point feature extraction operator ${ }^{[5]}$, it The amount of gray change can be expressed by an expression:

$$
\begin{aligned}
E_{x, y} & =\sum_{u, v} w_{u, v}\left[I_{x+u, y+v}-I_{u, v}\right]^{2} \\
& =\sum_{u, v} w_{u, v}\left[x X+y Y+o\left(x^{2}+y^{2}\right)\right]^{2}
\end{aligned}
$$

Among them: $E_{x, y}$ is the amount of gray change in the window; $w_{u, v}$ is a window function; $I$ is the gray function of the image. In order to improve the anti-noise ability of the algorithm, the Harris algorithm performs Gaussian smoothing on the image. The Gaussian window is the Gaussian function:

$$
w_{u, v}=\exp \left(-\left(u^{2}+v^{2}\right) / 2 \sigma^{2}\right)
$$

$\mathrm{X}$ and $\mathrm{Y}$ are first derivatives that can be approximated as:

$$
X=I \otimes(-1,0,1) \approx \partial I / \partial x, Y=I \otimes(-1,0,1)^{T} \approx \partial I / \partial y
$$

So $E_{x, y}$, can be expressed as:

$$
E_{x, y}=A x^{2}+2 C x y+b y^{2}
$$

Among them:

$$
A=X^{2} \otimes w, B=Y^{2} \otimes w, C=(X Y) \otimes w
$$

Harris proposed to distort $E_{x, y}$ :

$$
E_{x, y}=(x, y) M(x, y)^{T}
$$

Where $M$ is:

$$
M=\left[\begin{array}{ll}
A & C \\
C & B
\end{array}\right]
$$

For the region where there is a corner, the two eigenvalues of the matrix are the principal curvature of the autocorrelation function, so the two eigenvalues and the local region of the feature point are the central maxima, thus defining the corner response function, $R=\operatorname{det}(M)-k(\operatorname{trace}(M))^{2}$ Determine if the pixel is a corners. Where the value of $\operatorname{det}(M)=A B-C^{2}$, $\operatorname{trace}(M)=A+B$, and $k$ is a constant (the general $k$ has a value of 0.04--0.06) . 


\subsection{Improved Harris Corner Detection Algorithm}

In order to improve the efficiency of the algorithm, this paper proposes an improved algorithm to reduce the number of multiplication operations. So before selecting the corners, first extract the maximum value of the entire image, using $R=\operatorname{det}(M)-k(\operatorname{trace}(M))^{2}$ (the value of 0.04 ) formula, which can greatly reduce the image run time.

Improved algorithm description:

First, the image is converted into a grayscale image, the image $I_{x}$ direction and $I_{y}$ direction convolution, and summed up, this paper selects the $3 * 3$ convolution kernel. And then use the Gaussian filter on the $I_{x}^{2}$ and $I_{y}^{2}$ convolution, smoothing;

Second, using $R=\operatorname{det}(M)-k(\operatorname{trace}(M))^{2}$ pairs of the entire image to obtain the maximum value, get a matrix $R, R$ matrix $R=(1000 / \max (\max (R))){ }^{*} R$ processing, and then $R$ matrix median filter to get the matrix.

Third, Set the threshold value $T$ as a parameter with similar grayscale to determine, if the corresponding gray value of $M X$ does not change, and $R$ is greater than the set threshold, it is considered that the point is a gray point similar to the point (ie $(R==M X) \&(R>T)$ ), satisfy both condition can mark this point. The threshold $T$ is in the range of 20 to 30, and the value of $T$ can be adjusted according to the difference of the image. If the corner point is to be extracted, the threshold value $T$ is reduced, otherwise the threshold is reduced $T$, Which is the corner of the extraction, the edge of the image there will be a lot of pseudo-corner.

\section{NCC algorithm for corner rough matching}

There are three main methods of image matching based on grayscale, feature-based and transform-based domain. In this paper, NCC algorithm is used to normalize each other matching algorithm, which is based on the matching method of image gray information and has certain anti-noise capability.according to the corner of the field pixel gray similarity to match. The definition of NCC:

$$
C(p, d)=\frac{\sum_{(x, y) \in W_{p}}\left(I_{1}(x, y)-\overline{I_{1}}\left(p_{x}, p_{y}\right)\right)\left(I_{2}(x+d, y)-\overline{I_{2}}\left(p_{x}+d, p_{y}\right)\right)}{\sqrt{\sum_{(x, y) \in W_{p}}\left(I_{1}(x, y)-\overline{I_{1}}\left(p_{x}, p_{y}\right)\right)^{2} \sum_{(x, y) \in W_{p}}\left(I_{2}(x+d, y)-\overline{I_{2}}\left(p_{x}+d, p_{y}\right)\right)^{2}}}
$$

Where $I_{1}(x, y)$ and $I_{2}(x, y)$ are the gray scale values at the pixel at the image $I_{1}$ and the image $I_{2}$, respectively, where $p=\left(p_{x}, p_{y}\right)$ is the pixel-centered matching window to calculate the correlation coefficient, $C(p, d)$ is the calculated correlation coefficient, its value range is [-1,1], of course, the greater the value of the correlation coefficient is greater. In practical applications, when the correlation coefficient is less than a certain threshold, it is considered that the two corners are not correlated ${ }^{[6]}$.

In this article, the NCC matching algorithm has also been improved by the improved Harris algorithm to extract the corner, the first start of the original image to select a corner, select the order from top to bottom, from left to right, and then use the matching window In this paper, we use the 5 * 5 window) for each corner of contrast image , NCC match, this will come to a one-dimensional array, and then the array of ascending order, remove the last value that is the maximum, and set the threshold In contrast, the threshold set in this paper is 0.9. That is, with the largest correlation coefficient compared to if more than 0.9 that the two corners match, or that the two corners do not match. And then in turn remove the second corner of image $I_{1}$ and image $I_{2}$, the remaining corners to calculate the correlation coefficient, and so on to calculate the original image of all the corner correlation coefficient, In line with the conditions as a corner mark. So that the matching corner will be more accurate. Of course there will be some misplaced points here. 


\section{Experimental results}

The image size used is $340 * 458$, Fig. 2 is the matching of the two groups of different angles to be grayscale images.

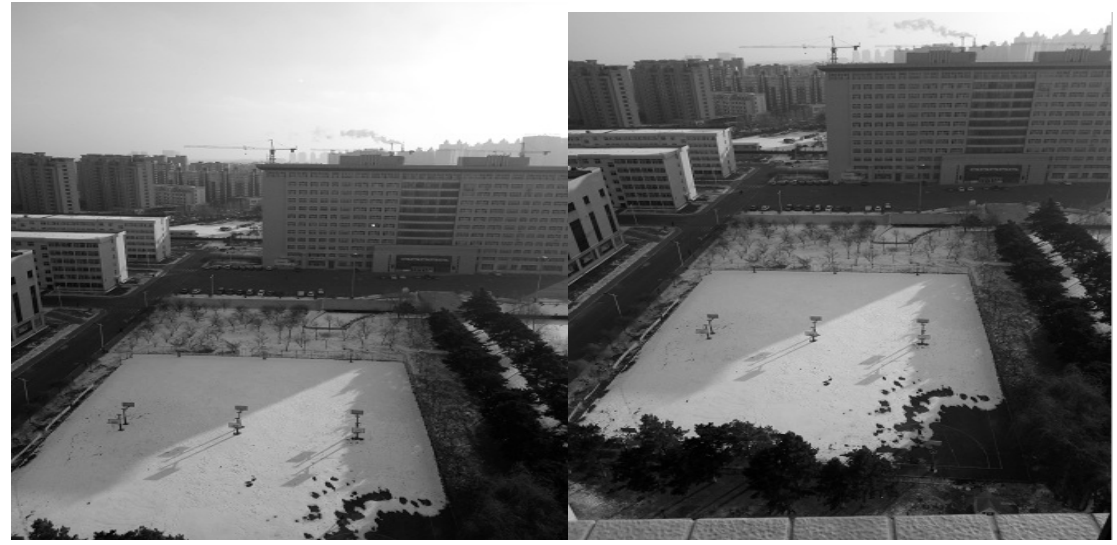

(a) original image

(b) contrast image

Fig.2 Two sets of original images to be matched

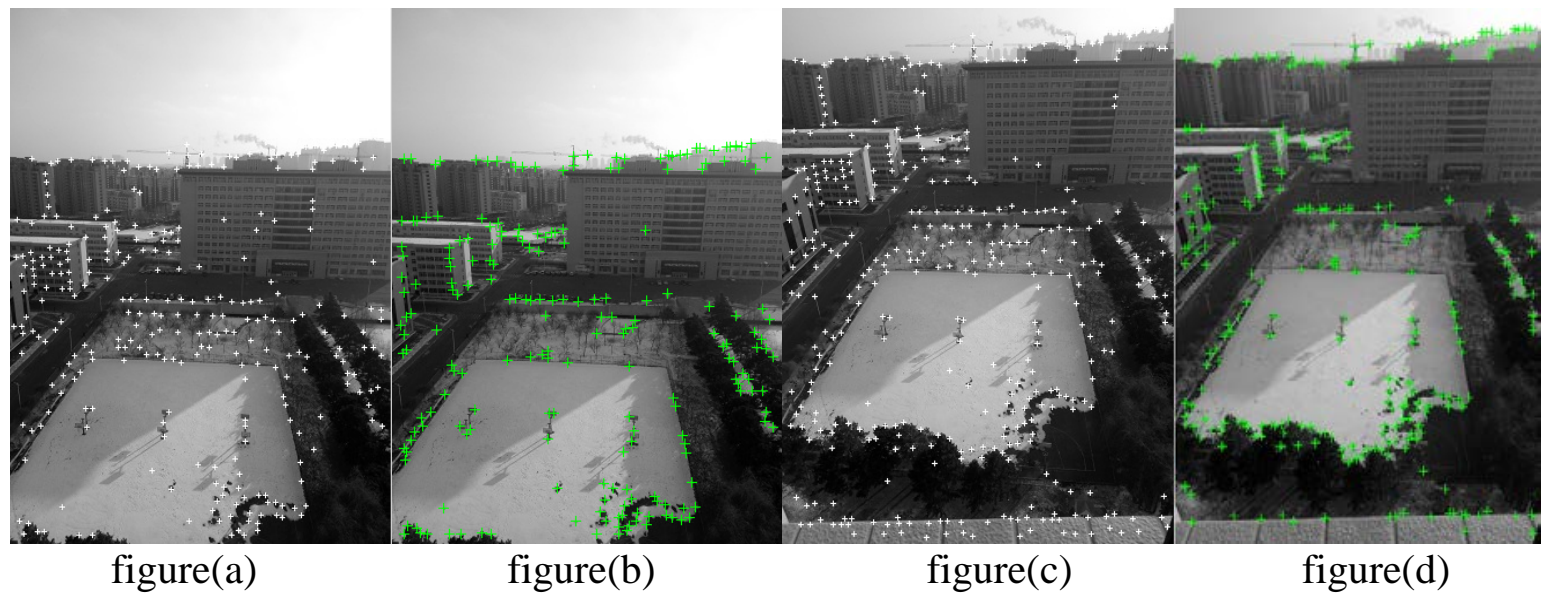

Fig.3 Corner extraction of two sets of images

Fig.3 is the use of improved algorithm and the classic Harris algorithm, respectively, the original image and contrast images for corner extraction ,figure (a) and figure(c) are the algorithm of this article. figure (b) and figure(d) are traditional algorithms

Table 1 The number of corner points and extract the corner time

\begin{tabular}{|c|c|c|c|c|}
\hline Algorithm & $\begin{array}{c}\text { a corner } \\
\text { point }\end{array}$ & $\begin{array}{c}\text { b corner } \\
\text { point }\end{array}$ & $\begin{array}{c}\text { figure a } \\
\text { extraction of } \\
\text { corner time }\end{array}$ & $\begin{array}{c}\text { figure b } \\
\text { extraction of } \\
\text { corner time }\end{array}$ \\
\hline $\begin{array}{c}\text { Traditional Harris } \\
\text { algorithm }\end{array}$ & 210 & 260 & $0.72753 \mathrm{~s}$ & $0.73271 \mathrm{~s}$ \\
\hline $\begin{array}{c}\text { Improve Harris } \\
\text { algorithm }\end{array}$ & 290 & 380 & $0.21859 \mathrm{~s}$ & $0.25562 \mathrm{~s}$ \\
\hline
\end{tabular}

From Table 1, we can see that the time of this algorithm is about $66 \%$ higher than that of the original algorithm. 


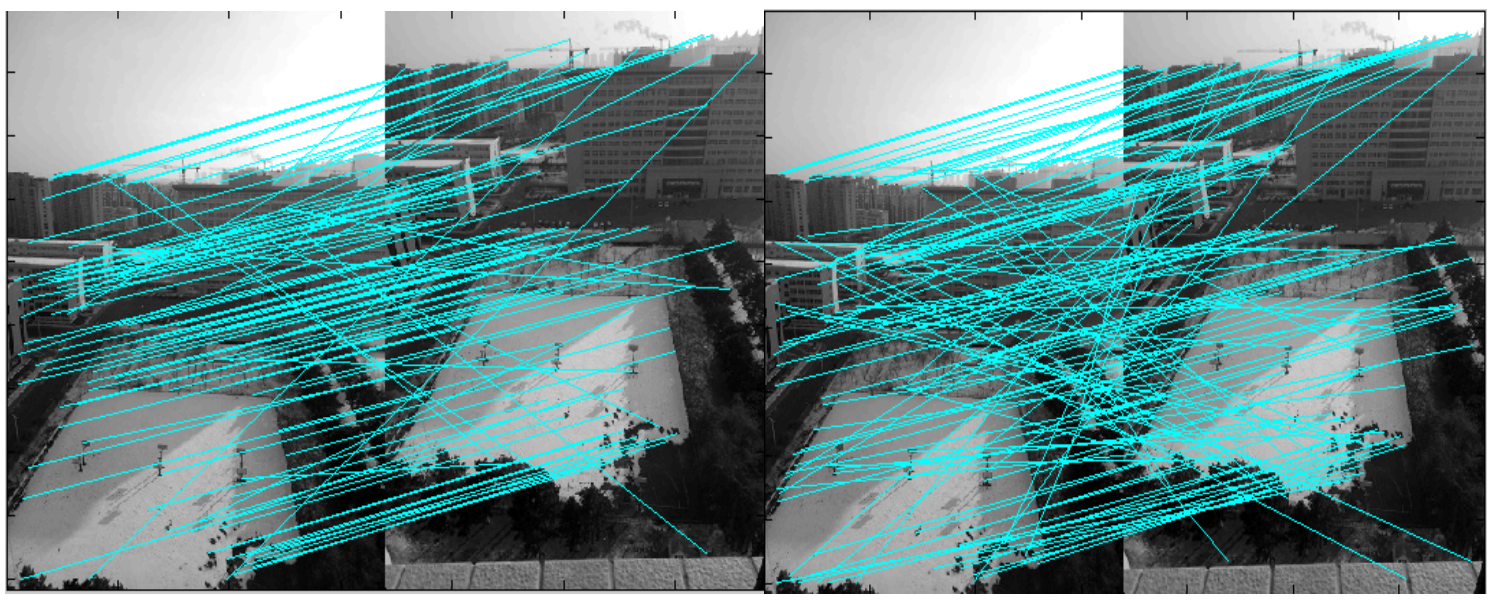

(a) This algorithm performs NCC matching

(b) Traditional Harris algorithm for NCC matching

Fig. 4 NCC algorithm corner rough match

Fig. 4 is the result of the rough matching of the corner points with the improved NCC algorithm and the original NCC algorithm, respectively

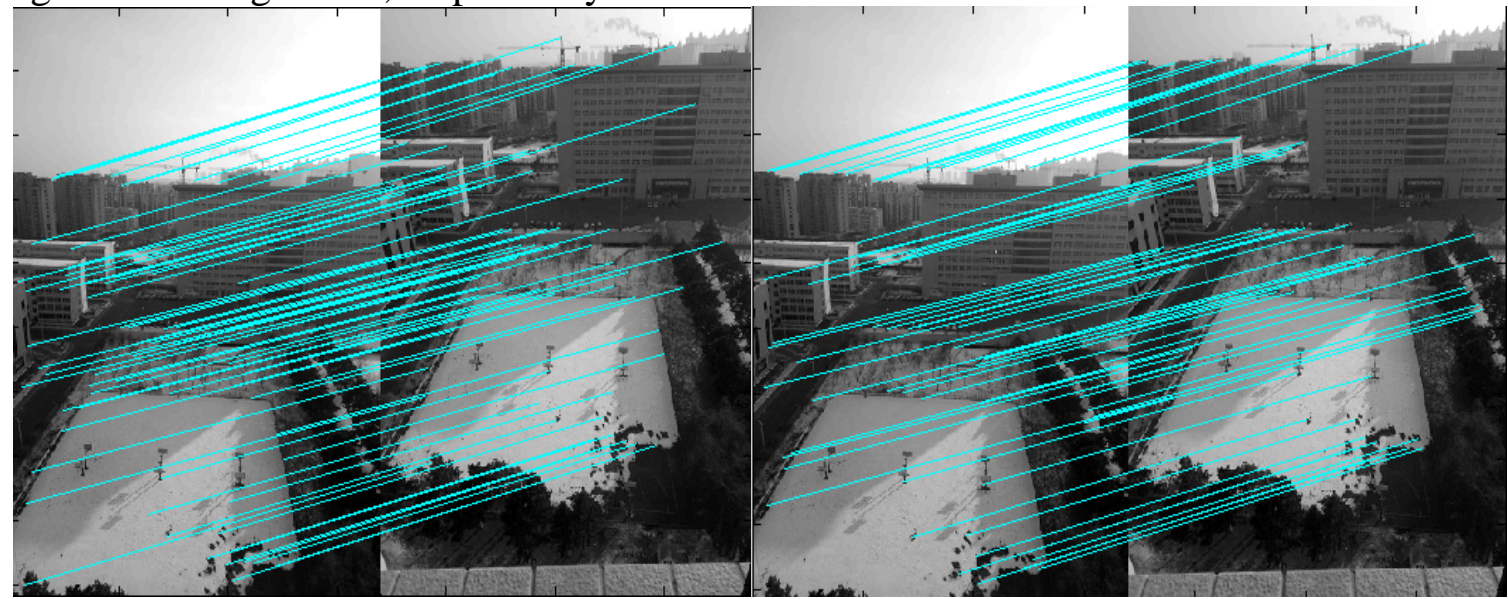

(a) This algorithm Ransac fine match

(b) Traditional Harris algorithm Ransac fine match

Fig. 5 Ransac algorithm corner point fine matching

Table 2 Experimental results statistics

\begin{tabular}{|c|c|c|c|c|c|}
\hline $\begin{array}{c}\text { Matching } \\
\text { algorithm }\end{array}$ & $\begin{array}{c}\text { NCC coarse } \\
\text { match }\end{array}$ & $\begin{array}{c}\text { Ransac fine } \\
\text { match }\end{array}$ & $\begin{array}{c}\text { Mistone } \\
\text { points }\end{array}$ & $\begin{array}{c}\text { Match } \\
\text { rate }\end{array}$ & Match time \\
\hline $\begin{array}{c}\text { Traditional } \\
\text { NCC match }\end{array}$ & 140 & 57 & 83 & $40.7 \%$ & $2.12171 \mathrm{~s}$ \\
\hline $\begin{array}{c}\text { Improved } \\
\text { NCC match }\end{array}$ & 100 & 73 & 27 & $73.0 \%$ & $4.36106 \mathrm{~s}$ \\
\hline
\end{tabular}

From Table 2 we can see that using this algorithm to improve the corner matching rate, the corner matching time has increased.

\section{Conclusions}

Although the traditional algorithm to extract the number of corners more, there are many pseudo-corner,so there are many mismatches when making a rough match, and the time to match the search is increased. This algorithm can extract corner points quickly, and it can reduce the number of false matches in NCC matching, and improve the image matching rate. The experients show that this algorithm is an effective corner matching algorithm. Although the algorithm has less time in extracting corners, the accuracy of corner detection has not been considered more finely. 
Therefore, there is still some improvement in the accuracy of corner extraction, and the time of rough matching is also expanded about twice as much, it is also necessary to improve the place.

\section{References}

[1] Qi Jun, Tian Yimin, Wang Xin.An improved Harris corner detection algorithm [C]. Journal of Beijing Institute of Graphic Communication, 2014.

[2] S Hen H T, Hu W C. A Rotationally Invariant Two-phase Seheme for Corner Detection [J]. Pattern Recognition, 1996, 29 (5): 819-828.

[3] Gong Ping, Liu Xiangbin, Zhou Peng.An improved Harris corner detection algorithm. Computer Engineering and Applications, 2010,46 (11): 173-175..

[4] Robert M. The topographic primal sketch [J]. The International Journal of Robotics Research, 1983, 2 (1): 21-24.

[5] Harris C G Stephens M J. A Combined Comer and Edge Detector [C] // Prcoceeding of the 4th Alvey Vision Conference. Manchester, England: [s.n.], 1988.

[6] Xue Fei, ZHANG Rong-guo, ZHANG Jianguo, CHEN Da-chuan. Fast characteristics based on NCC and SSDA Point matching fusion algorithm [J] .Computer \& Digital Engineering, 2010,10 (19): 38-42. 